\title{
UNE CAMPAGNE DE MESURES OBJECTIVES ET PERCEPTIVES EN ACOUSTIQUE DES SALLES
}

\author{
O. WARUSFEL et J.P. JULLIEN \\ IRCAM, 31 nue Saint-Meni, F-75004 Paris, France
}

\begin{abstract}
RESUAME - Cet article est consacré a la description d'une campagne de mesures et d'écoutes dans le domaine de racoustique des salles. Le but de $c$ travail est d'une part de valider un emvironnement informatique de modélisation acoustique développé a l'IRCAM et d'autre part de préciser les connaissances sur la perception de la qualité acoustique des salles. L'article décrit les principes qui ont présidé à l'élaboration de cotte base de données.
\end{abstract}

\begin{abstract}
This paper describes the organization of a data base in the field of room acoustics. This data base consists of objective measurements recorded in various concert hals together with perceptive judgments collected in the same halls during concerts. The aim is to validate a computer environnement dedicated to room acoustics prediction that was developped at IRCAM and to improve our knowledge on perceptual aspects linked to the acoustical quality of a room.
\end{abstract}

\section{INTRODUCTION}

Le laboratoire d'acoustique des salles de l'IRCAM a organisé au début de l'année 1989, dans le cadre d'une commande du Ministère de la Culture, une campagne de mesures dans différentes salles parisiennes et européennes. Ce travail fait suite à un ensemble d'études effectuées dans différents domaines de l'acoustique des salles. En acoustique prévisionnelle le laboratoire a introduit des techniques spécifiques de prédiction intégrant différents modèles physiques de la propagation acoustique. Dans le domaine de l'analyse perceptive I'IRCAM a mené une étude sur les relations entre les critères acoustiques et notre perception.

L'intérét de cette campagne est d'une part de recueillir une base de données permettant une confrontation systématique des résultats estimés par les outils de modélisation et d'autre part de vérifier nos connaissances fondamentales sur la perception auditive de la qualité acoustique des salles. En effet le succès d'un environnement de modélisation informatique dépend non seulement de la précision des modèles utilisés mais également de la pertinence perceptive des résultats objectifs obtenus par les simulations.

\section{'2TRAVAUX ANTERIEURS}

\subsection{Modélisation informatique}

L'IRCAM a développé un environnement de simulation informatique qui combine différents modèles physiques de la propagation acoustique : modèle en ondes planes et réflexions spéculaires, modèle de diffraction, modèle de réflexion diffuse. Le choix de combiner ces modèles a été dicté par un travail préalable qui a montré leur complémentarité pour l'estimation des différentes grandeurs liées à la qualité acoustique de la salle. Les caractéristiques et performances principales de ces modèles sont rappelées ci-dessous.

Modèle en ondes planes Méthode des cónes : les développements effectués par l'IRCAM autour de ce modèle, courramment utilisé dans les logiciels de simulation, ont eu pour objectif de pallier certaines de ses faiblesses. En particulier lindétermination sur l'énergie et le temps d'arrivée des toutes premières réflexions, inhérente à la discrétisation du rayonnement de la source sous forme de rayons, a été corrigée en combinant la méthode des cónes à celle, exacte, des images pour les 2 premiers ordres de réflexion. Par sa nature, ce modẻle foumit de très bons résultats pour l'estimation de l'organisation temporelle précoce.

Modelle de diffraction: en acoustique des salles, le plus souvent, les exigences de visibilité de la scène par les auditeurs suffisent à éviter les phénomènes de masquage. Cependant il existe une situation traditionnelle pour laquelle des masquages importants ont lieu : la fosse d'orchestre et les loges d'Opéra. En présence de surfaces masquantes les modèles de propagation spéculaire ou diffuse vont réfléchir l'énergie dans le demi-espace incident, considérant ainsi qu'il y a masquage complet du demi-espace arrière. Si cette approximation est acceptable pour le comportement tardif de la salle en revanche limportance de l'onde directe pour la perception a motivé l'enrichissement de la description de son masquage par l'introduction d'un modẻle de diffraction.

Modele markovien de la diffusion : Afin de rendre compte du caractère non parfaitement spéculaire des réflexions, le modèle précédent est couplé à une méthode basée sur l'hypothèse de réflexion diffuse (loi de Lambert). La répartition entre les deux modèles est gérée à chaque réflexion par lintermédjaire d'un coefficient dépendant de la nature des parois. La propagation de l'énergie diffuse est réalisée par une méthode dite markovienne. Les propriétés de cette description markovienne permettent notamment d'accéder de manière 
elficace aux critères liés au régime tardif de la salle (durée et niveau de réverbération). En décrivant de manière individuelle les couplages géométriques et acoustiques entre les différentes parois cette méthode permet une estimation du comportement stationnaire de la salle plus riche que les approches globales de Sabine ou Eyring. En particulier elle permet d'accéder à la répartition spatiale de l'énergie du champ réverbéré.

\subsection{Caractérisation perceptive}

Les études menées à l'IRCAM dans le domaine de la perception ont pour but une représentation objectivable des divers aspects qui gouvernent notre perception. Ces relations représentent un double intérêt : permettre une interprétation perceptive à lappui d'un diagnostic acoustique et autoriser un contrôle, pertinent pour la perception, d'espaces a acoustique variable [1]. Lors des programmes de recherche antérieurs ces relations ont été établies à partir de tests psycho-expérimentaux réalisés dans des espaces acoustiques simulés (hémisphère de hauts-parleurs en chambre sourde). L'un des principaux objets de la campagne est par conséquent de tester la pertinence de ces relations en situation de concert. D'autre part l'intention est de compléter cefte approche descriptive de la perception par une analyse des préférences.

\section{Remarques préliminaires}

L'un des principaux problèmes liés à l'évaluation perceptive en situation de concert est le choix du questionnaire proposé aux auditeurs. Différentes stratégies peuvent être adoptées : questionnaires basés sur des échelles descriptives ou sur des échelles de préférence. Des études précédentes ont montré que les analyses effectuées à partir de jugements exclusits de préférence dépendent fortement du choix des configurations testées. En revanche le recours à des échelles descriptives pose le problème de la redondance des échelles proposées et de leur acception sémantique par les différents auditeurs. Les études de laboratoire effectuées à l'IRCAM, qui sont à l'origine du questionnaire proposé ici, ont tenté de limiter ces inconvénients. Des situations d'écoute simulées étaient comparées deux à deux, les sujets ne s'exprimaient que sur les différences perçues. De ces données, analysées par la méthode INDSCAL, a été déduite une structure perceptive constituée de dimensions communes à tous les sujets et appelées facteurs perceptits. Chaque facteur a pu être relié quantitativement à un jeu de critères acoustiques. C'est sur la base de ces facteurs, nommés a posteriori par le laboratoire, qu'a été construit le questionnaire de la campagne d'écoute. Bien que les problèmes sémantiques ne soient pas complètement évacués, ce type d'analyse a permis d'assurer l'indépendance et l'exhaustivité du jeu de facteurs sur l'ensemble des tests psycho-expérimentaux. Ces facteurs, ainsi que leur correspondances objectives, sont présentés dans le tableau ci-dessous. Pour les définitions des critères objectifs on se reportera aux références suivantes [2].

\begin{tabular}{|c|c|c|c|c|c|}
\hline FACTEURS & $\begin{array}{l}\text { CORRESPONDANCE } \\
\text { OB.JECTVE }\end{array}$ & NOM & FACTEURS & $\begin{array}{l}\text { CORRESPONDANCE } \\
\text { OBJECTIVE }\end{array}$ & NOM \\
\hline F1 & Clarté a $80 \mathrm{~ms}(\mathrm{C} 80)$ & Précision d'écoute & F7 & Rapport Son direct/1ère rêt. (Dir/Ref) & Contraste \\
\hline F2 & C80, Son direct étendu (DirE) & Distance subjective & $\mathbf{F 8}$ & Barycentre temporel des 1 ère réfl. & Fluidité \\
\hline F3 & $\mathrm{C} 80$, niveau sonore $(\mathrm{G})$ & Presence & $\mathrm{Fg}$ & Différence G grave/medium & Chaleur \\
\hline F4 & Temps de réverberation (Tr), G & Réverberance & F10 & Différence $\mathrm{G}$ aigus/médium & Brillance \\
\hline F5 & Barycentre spatial des 1ère réft. & Localisation & $\mathbf{F} 11$ & Gradient du Tr grave/médium & intimité \\
\hline F6 & Efticacité latérale (LE) & Enveloppement sonore & F12 & Gradient du Tr aigus/medium & vivacité \\
\hline
\end{tabular}

Tableau 1. Facteurs perceptifs et correspondances objectives Table 1. Perceptual factors and their links with objective criteria

\section{DESCRIPTION DE LA BASE DE DONNEES}

L'élaboration de la base de données doit répondre à différents niveaux de contraintes. Citons, pour les analyses psychoacoustiques, le respect de certains paramètres statistiques (nombre de stimuli, nombre de sujets...) ou dans le domaine de la caractérisation objective l'assurance de conditions de mesures reproductibles. D'autre part le protocole doit être choisi en fonction des axes d'analyse envisagés. Les paragraphes suivants décrivent les critères qui ont été retenus pour construire la base de données.

\subsection{Sélection des salles}

Les salles retenues sont regroupées ci-dessous en fonction de leur vocation. Le théâtre des Champs Elysées apparaît dans deux catégories correspondant à deux configurations scénographiques étudiées. Le choix des salles a été guidé par les critères suivants :

_variation du parti architectural: Différentes formes de salle ont été choisies afin de valider les programmes de simulation pour des configurations géométriques variées (volume, présence de balcons ou de fosse...). 
présence de salles réputées : afin d'engager une étude sur la prétérence.

présence de salles de conception récente : permettant la caractérisation acoustique de formes ou matériaux contemporains.

\begin{abstract}
Auditorium
Musee du Louvre

Muse dorsay
\end{abstract}

\author{
Salle de concert \\ Concertgebouw d'Amsterdam \\ Musikverein de Vienne \\ Philharmonie de Berlin \\ Pleyel \\ Théatre des Champs Elysés
}

\author{
Opéra \\ Garnier \\ Scala de Milan \\ Theatre des Champs Elysees
}

Tableau 2. Liste des salles retenues pour la campagne de mesures et d'écoutes

Table 2. List of selected halls for the objective and perceptive data base

\subsection{Mesures objectives}

De manière à conduire des comparaisons rigoureuses entre les salles un protocole expérimental a été adopté pour l'ensemble de la campagne. Celui-ci répond aux points suivants:

_Répartition spatiale des sources et des récepteurs : le travail de caractérisation objective des salles est mené à partir de critères déduits des réponses impulsionnelles. Afin d'analyser la dépendance de la qualité acoustique en fonction de la position du récepteur et de la source, un balisage spatial systématique a été appliqué à toutes les salies (figure 1). Les réponses impulsionnelles sont mesurées pour chaque couple source/récepteur. De manière à caractériser également l'écoute réciproque des instrumentistes les points récepteurs ont été étendus aux zones scéniques (plateau el losse). Le pas du réseau spatial a été déterminé en fonction de l'ordre de grandeur des critères temporels utilisés en acoustique, de la surface des salles et de leur complexité géométrique. Les salles comportant un axe de symétrie, le balisage de la zone "auditoire" a été réduit à la moitié gauche. Des vérifications de cette hypothèse ont été effectuées sur quelques points récepteurs.

_de manière à traduire différents aspects de la perception, certains critères font intervenir des pondérations spatiales et temporelles des réflexions. Pour ces critères les mesures doivent être effectuées avec différentes sensibilités microphoniques : omni, cardio, "figure 8 ".

les particularités de directivité des sources instrumentales privilégient l'excitation de certaines parois de la salle. Aussi plusieurs orientations de la source ont été systématiquement utilisées : face, "cour", "jardin", plafond et arrière. Les caractéristiques de la source sonore et des récepteurs ont été déterminées de façon précise en salle anéchoïque.

\subsection{Tests perceptifs}

Les réponses des auditeurs ont été recueillies, en situation de concert, sur la base d'un questionnaire structuré . Ce questionnaire comprend une première partie destinée à la description de la perception. Celle-ci reprend les échelles perceptives exposées précédemment auxquelles ont été ajoutés quelques aspects mentionnés dans la littérature (dimension subjective de la salle, dynamique, balance orchestrale). Le nombre de degrés présentés sur chaque échelle a été déduit des seuils perceptifs notés au cours des études précédentes. Ces échelles descriptives sont réparties en deux groupes : d'une part les facteurs liés à la perception globale de l'effet de salle (réverbérance, niveau, coloration...) et d'autre part ceux liés à la perception des instruments (précision, distance subjective, contraste....). Pour ces derniers il est proposé au sujet de s'exprimer sur la perception globale de l'effectif instnumental el de manière détaillée par pupitre. L'auditeur est également invité à indiquer la présence éventuelle de défauts correspondant à des excès sur les facteurs de coloration (lourd, agressif ...). Une deuxième partie est consacrée aux jugements d'appréciation subjective : impression générale, adaptation de la salle à l'oeuvre, préférence inter-places. Enfin sont demandées les appréciations sur l'interprétation et l'oeuvre de manière à déceler la dépendance des réponses avec le contexte musical. Quelques échelles extraites de ce questionnaire sont présentées ci-dessous

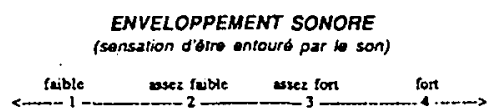

REVERBERANCE

(ettet os trainge du son)

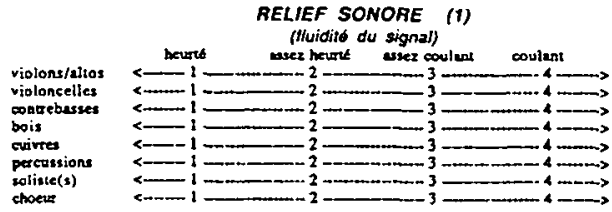




\section{Protocole des tests perceptifs}

En toute rigueur les tests devraient proposer à une méme population d'écouter à toutes les places et dans toutes les salles les mêmes oeuvres musicales. Cette procédure est naturellement dífficile à réaliser, par conséquent il a été choisi de croiser paramètres constants et variables. Pour cela deux groupes dauditeurs ont été constitués : un groupe d'auditeurs stable et un groupe renouvelable. De mérme, suivant les concerts, les auditeurs sont invités à juger les mêmes ceuvres en différentes places de la salles, ou différentes oeuvres à une méme place. Pour cela, ef suivant les contraintes de programmation, les tests sont organisés, pour chaque salle, sur deux concerts consécutif́s proposant le même programme musical. Les sujets occupent à tour de rôle différentes places correspondant aux positions réceptrices de la campagne de mesures.

\subsection{Simulations}

Pour le travail de validation des programmes de simulation la base de données a été complétée par la discrétisation informatique des différentes salles à partir des plans architecturaux (figure 2). Ce travail consiste à décrire sous forme de facettes planes la géométrie de la salle. Chaque face est caractérisée par un coefficient d'absorption et un coefficient de diffusion dépendant du matériau. Les récepteurs et les sources sont disposés suivant la grille spatiale utilisée lors des mesures. La directivité de la source ainsi que celles des différents types de microphones sont prises en compte par des modéles respectant les caractéristiques mesurées des transducteurs utilisés pour les mesures.

\begin{tabular}{|c|c|c|c|c|c|}
\hline SALLE & MESURES OBJECTIVES & TE & S PERCEP & JIFS & SIMULATION \\
\hline & $\begin{array}{l}\text { Nbre de couples source/rópt } \\
\text { I nbre de meaures }\end{array}$ & $\begin{array}{l}\text { Nbere } \\
\text { doourres }\end{array}$ & $\begin{array}{l}\text { Nbre } \\
\text { orauditours }\end{array}$ & $\begin{array}{l}\text { Nbre de places } \\
\text { par auditeur }\end{array}$ & Nbre de surfaces \\
\hline ConcontGoboun & 341 couples / 630 mesures & 3 & 9 & 4 & 234 \\
\hline $\begin{array}{l}\text { Philharmonie } \\
\text { Musikverein }\end{array}$ & $\begin{array}{l}484 \text { couples / } 919 \text { mesures } \\
300 \text { couples / } 600 \text { mesures }\end{array}$ & 3 & $\begin{array}{l}12 \\
\text { non realises }\end{array}$ & 4 & $\begin{array}{l}\text { non discrétsée } \\
220\end{array}$ \\
\hline Scala da Milano & 361 couples / 764 mesures & 1 & 12 & 4 & non achevee \\
\hline Pleyel & 420 couples / 816 mesures & 5 & 12 & 4 & non achevée \\
\hline Orsay & 89 couples / 177 mesures & 3 & 6 & 3 & non achevee \\
\hline Louvie & 95 couples / 191 mesures & 4 & 6 & 6 & 200 \\
\hline Champs Elyseos & s 419 couples / 881 mosures & 1 & $\bullet$ & 6 & 470 \\
\hline
\end{tabular}

Tableau 3. Tableau récapitulatif des mesures et écoutes constituant la base de données Table 3. Number of measurements and perceptive tests of the data base

\section{PERSPECTIVES D'EXPLOITATION}

La somme des données contenues dans cette base autorisera, outre une analyse critique des travaux antérieurs, des axes multiples d'analyse. Citons, dans le domaine de la propagation acoustique dans les salles, l'étude de la distribution spatiale du champ réverbéré, l'analyse des problèmes de couplage entre les volumes scène, fosse et auditoire ou le comportement local de certains matériaux (diffusion). Dans le domaine de la perception, sont en cours d'analyse les relations entre les différents aspects de la perception et les jugements de préférence ainsi que les problèmes de dépendance entre te contexte musical (oeuvre, effectif, formation instrumentale) et la qualité acoustique perçue par les auditeurs.

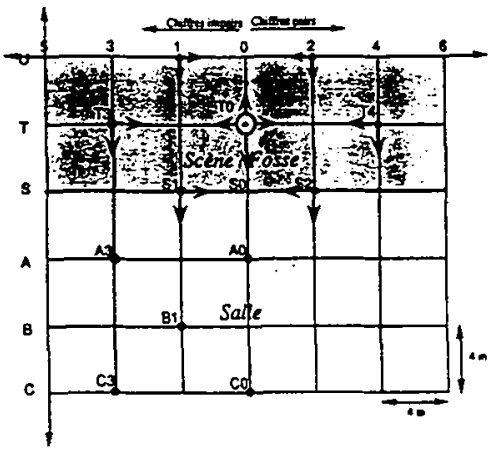

Figure 1. Gritle spatiale de disposition et d'orientation des sources et reopteurs Figure 1. Spatial disposition and orientation of sources and receivers

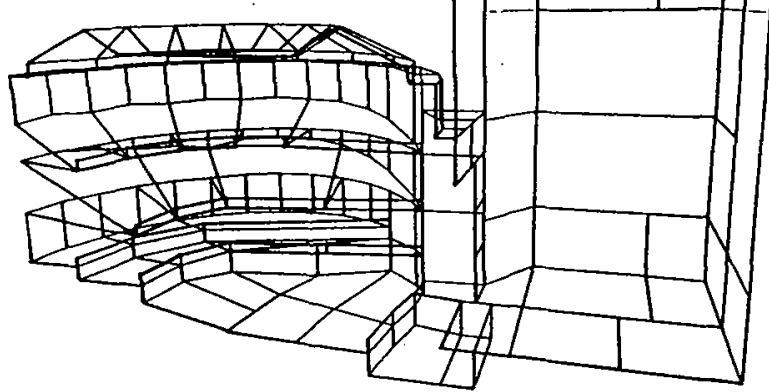

Figure 2. Theatre des Champs Elysees Vue du modile / View of the model

[1] G. Bloch et J.P. Jullien Commande perceptive dun systeme à acoustique variable _ 1er CFA Lyon_France, Avril 1980

[2] J.P. Jullien, C. Lavandier, O. Warusfel_C Compared analysis of the clarity and the reverberation in room acoustics_Proceedings of 13th ICA, Belgrade, Ao0t 1989. 